\title{
The management of pretibial lacerations
}

\author{
P Singh, M Khatib, A Elfaki, N Hachach-Haram, E Singh, D Wallace
}

Department of Plastic and Reconstructive Surgery, Addenbrooke's Hospital, University of Cambridge, Cambridge, UK

ABSTRACT

INTRODUCTION Pretibial lacerations are common injuries, often presenting in the elderly and infirm. Unclear management pathways often result in inappropriate care. We identify patient demographics, morbidity risk factors, injury severity and management options.

MATERIALS AND METHODS This retrospective study involved analysing databases and hardcopy notes for patients admitted with pretibial lacerations to Addenbrooke's Hospital, January to December 2012. Microsoft Excel and Fishers exact test were used to analyse the data with a $P$-value of less than 0.05 representative of statistical significance. Information on patient demographics, site of lesion, preoperative symptoms, management, operative details and clinical outcomes were collected.

RESULTS A total of 36 patients were identified; the mean age was 79 years ( \pm 16 years, 1 standard deviation) with a three to two female to male preponderance; $57 \%$ of injuries were caused by mechanical fall, $33 \%$ traumatic blunt impact and $7 \%$ road traffic accidents. American Society of Anesthesiologists physical status classification was $43 \%$ level III, 40\% II, 9\% I and 9\% IV. Dunkin classification of severity was $33 \%$ grade III, $30 \%$ grade I, $24 \%$ grade IV and $12 \%$ grade II. Median inpatient duration was 11 days for surgically managed compared with 15 days for conservatively managed patients.

DISCUSSION Pretibial lacerations tend to affect the elderly. Management is compounded by polypharmacy and comorbidities. If inadequately managed, such injuries can adopt characteristics of chronic wounds, with lengthy inpatient stays. Surgical intervention may be appropriate where injuries are severe and the patient stable enough for theatre.

CONCLUSIONS We believe that surgical management with autologous tissue repair, with minimal delay between presentation and theatre, is warranted for extensive injuries wherever possible, with conservative management used for predominantly less extensive pretibial lacerations.

\section{KEYWORDS}

Pretibial lacerations - Skin graft - Debridement

Accepted 11 June 2017

CORRESPONDENCE TO

Prateush Singh, E: singh.prateush@gmail.com

\section{Introduction}

Pretibial lacerations are common yet under-appreciated injuries. They frequently present to plastic surgery, emergency and orthopaedic departments but no agreed care pathway currently exists. They are of pressing concern in the elderly and infirm owing to decreased dermal collagen integrity resulting in friable skin, which exacerbates injury from trivial trauma. ${ }^{1}$ Underlying haematomas often co-exist and are potentiated by anticoagulant medications such as warfarin, ${ }^{2}$ which compromises surrounding healthy tissue by inducing local tissue ischemia via pressure effects and the production of reactive free radicals. ${ }^{3}$ Many underlying comorbidities such as diabetes mellitus, peripheral oedema, peripheral vascular disease and corticosteroid use have all been associated with decreased skin integrity through oxidative stress and matrix-metalloproteinase mechanisms. ${ }^{4}$ Malnutrition further compromises skin durability and adequate wound healing. ${ }^{5}$ With trauma being a leading cause of pretibial lacerations, the increased incidence of visual impairment and diminishing mobility in the elderly population makes them particularly vulnerable. ${ }^{6}$

The current incidence of pretibial lacerations presenting to accident and emergency departments is 5.2 per 1000 per year in the UK and with an ageing population this is likely to increase. ${ }^{7}$ The financial impact is significant, with an esti-

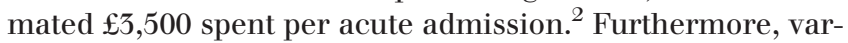
iations in management in primary and secondary care have led to complicated and compromised clinical outcomes, with many patients going on to require plastic surgery and tissue viability services, wound debridement, grafting and negative-pressure dressings. This negatively impacts on patient morbidity. ${ }^{8}$

We aimed to identify the demographics and epidemiology of pretibial lacerations in patients attending a tertiary trauma centre, morbidity risk factors and management strategies in often elderly patient with multiple comorbidities and polypharmacy. Management of pretibial lacerations is dependent on the extent of injury, which can be classified according to the Dunkin classification (Table 1). ${ }^{9}$ 


\section{Materials and Methods}

In this retrospective study, patient databases, theatre lists and hardcopy notes were reviewed for patients admitted with pretibial lacerations to Addenbrooke's Hospital, Cambridge, over a 1-year period from January to December 2012. Patient demographics, site of lesion, preoperative symptoms, imaging, management, operative details and clinical outcome data were collected. Lacerations not isolated to the pretibial region were excluded.

Statistical analysis was performed using Microsoft Excel with Fisher's exact test used for categorical data. Analysed data were described with mean and median values accompanied by one standard deviation (SD) distribution.

\section{Results}

A total of 36 patients with 36 pretibial lacerations presented to Addenbrooke's Hospital between January and December 2012. The mean age was 79 years $( \pm 16$ years, 1 SD) with a three to two female to male preponderance. Injuries were caused by mechanical fall in $57 \%$ of cases, traumatic blunt impact in $33 \%$ and road traffic accidents in $7 \%$.

Evaluation of medical history revealed two patients with more than three comorbidities. According to the American Society of Anesthesiologists (ASA) physical status classification, $43 \%$ were level III, $40 \%$ level II, $9 \%$ I and $9 \%$ IV. The extent of pretibial injury was Dunkin grade III in 33\%, grade I in $30 \%$, grade IV in $24 \%$ and grade II in $12 \%$ (Table 1).

Assessment of medication revealed that $31 \%$ were taking corticosteroids, $56 \%$ aspirin, $8 \%$ clopidogrel, $22 \%$ warfarin and $25 \%$ aspirin and warfarin together. The mean length of inpatient stay was 18 days ( \pm 26 days, $1 \mathrm{SD}$ ), subdivided into 11 days ( \pm 12 days, 1 SD) for surgically managed patients and 62 days ( \pm 79 days, 1SD) for conservatively managed patients. The data were skewed significantly because three conservatively managed patients stayed for 190, 160 and 44 days, respectively, far out of the normal range of remaining inpatient duration. Analysis of the median length of stay revealed a surgical inpatient duration of 11 days and conservative inpatient duration of 15 days.

In terms of surgical intervention, debridement and reconstruction with split thickness skin grafting was done in $61 \%$ of surgically managed cases with graft take at $94 \%$ ( $\pm 13 \%$, 1SD) on first attempt. Patients receiving grafting stayed an average of 12 days ( \pm 13 days, 1SD), far lower than conservatively managed patients. The 1-year mortality observed was $22 \%$, due to other medical complications and concomitant illnesses.

\section{Discussion}

We evaluated our experience of managing pretibial lacerations over a 1-year period. Our patient population was consistent with the literature: the average age was 79 years, predominantly female $(67 \%)$ with significant comorbidities ( $43 \%$ classified level III and $40 \%$ level II by the ASA physical status classification)..$^{10}$
In our study, 33\% were classified as Dunkin grade III and $24 \%$ grade IV. Of the total, 23 patients were managed operatively, requiring an inpatient stay of 11 days ( \pm 12 days $1 \mathrm{SD})$, whereas 13 patients were managed conservatively, resulting in an extended inpatient stay of 62 days $( \pm 78$ days 1 SD) when including a small number of patients staying for a disproportionately long time. With such outliers excluded, the conservative length of stay was 15 days. We note these three outliers, as they illustrate that conservative management has the propensity to cause lengthy inpatient durations and highlight the multidisciplinary nature of dealing with such injuries as increased physiotherapy, occupational therapy and community care packages were required before these patients could be safely discharged. This increase in inpatient stay for conservatively managed patients is consistent with Cahill et al., ${ }^{7}$ who found that surgically managed patients stayed on average 59.8 days compared with 123 days for those conservatively managed, and TubokuMetzger et al., ${ }^{11}$ who found that surgically managed patients stayed 55 days compared with 110 days for those managed conservatively.

Pretibial injuries can take on characteristics of chronic wounds that are difficult to heal given the increased patient age, underlying comorbidities such as diabetes, peripheral vascular disease, venous hypertension, cardiac failure and renal failure, and given the poor vascularisation of this region. Drugs such as warfarin, corticosteroids and aspirin potentiate haematoma formation which complicates management and may need to be dealt with independently. ${ }^{12}$ Shankar et $a l .{ }^{13}$ report graft take for pretibial injuries at $100 \%$ but do not consider patient comorbidities or grade of pretibial injury, which could result in graft failure. Patients with reduced graft take in our study were ASA IV, Dunkin grade III or IV elderly patients on anticoagulation. Graft take was assessed by consultant plastic surgeons and photographic evidence taken. Patients with failed grafts underwent subsequent debridement and were regrafted with $100 \%$ take on subsequent attempts. Although Shankar et $a l .{ }^{13}$ encourage immediate discharge, we believe that in an elderly population with potentially multiple comorbidities, Dunkin III and IV patients with skin grafts should be monitored for graft take, wound healing, mobilisation under physiotherapy supervision and occupational therapy, and and should have a social package assessment before discharge where appropriate, all of which contribute to inpatient duration.

Evidence suggests that there is a clear benefit from early mobilisation rather than bed rest in grafted pretibial lacerations with no decrease in the extent of graft take. ${ }^{14}$ Inpatient stay is consequently decreased, together with its associated morbidity. In the financial strains of today's economic environment, one must also be aware of the financial benefits of saving bed days per patient, an average of 4 days saved per patient at our institution.

Conservative management may be instigated for high ASA patients who are deemed unsuitable for surgery but, wherever possible, delay between injury and surgery should be minimised. Patients with pretibial lacerations are often 
Table 1 Dunkin classification of pretibial injuries with recommended management

\begin{tabular}{|c|c|c|c|}
\hline Type & Appearance & Description & Recommended management \\
\hline I & & Lacerations & $\begin{array}{l}\text { Clean with saline } \\
\text { SteriStrip } \\
\text { Supportive dressing }\end{array}$ \\
\hline II & & Laceration or flap with minimal haematoma and/or skin-edge necrosis & $\begin{array}{l}\text { Clean with saline } \\
\text { Debride non-viable tissue } \\
\text { Evacuate haematoma } \\
\text { SteriStrip }^{\text {TM }} \text { without tension } \\
\text { Supportive dressing }\end{array}$ \\
\hline III & & Laceration or flap with moderate to severe haematoma and/or necrosis & $\begin{array}{l}\text { Debride non-viable tissue } \\
\text { Split-skin graft under anaesthesia }\end{array}$ \\
\hline IV & & Major degloving injury & $\begin{array}{l}\text { Debride non-viable tissue } \\
\text { Reconstruct under general anaesthesia }\end{array}$ \\
\hline
\end{tabular}

neglected and postponed on surgical theatre lists, prolonging starvation times and contributing to malnutrition in patients with often multiple comorbidities and frailty. ${ }^{15} \mathrm{In}$ addition, there is evidence to suggest that free radicals produced by iron from haemoglobin breakdown in associated haematoma contributes to surrounding tissue damage and wound morbidity. ${ }^{3}$ A prospective study on the surgical management of pretibial lacerations reported a cancellation rate of $50 \%$, overall starvation time of 18 hours and a delay between referral and surgery of 10 days. ${ }^{15,16}$ Tubuku- 
Metzger et al. ${ }^{11}$ reviewed all patients admitted to their tertiary plastic surgery centre and found that delay in surgery had significant effects on outcome. These figures must be reduced if patient outcomes are to be improved.

Our recorded 1-year mortality was 8 of 36 patients but the Office for National Statistics (England and Wales) reported an all-cause mortality for females aged 81 years-old of 47 per 1000 in 2012 and for those aged 80-84 years of 53.3 per 1000 in 2013, but the rate for the pretibial population was more than double these values at 6 -month follow up. ${ }^{17}$ The impact of pretibial injuries on morbidity is significant and their incidence is likely to increase as the population ages. The need for a strategic management algorithm with multidisciplinary input is necessary if improvements in patient care and outcome are to be achieved.

Our study is limited by its retrospective nature and as we were only able to capture a few cases. In addition, although patient databases were searched using surgical codes to identify appropriate cases, some cases could have been missed due to inaccurate coding.

\section{Conclusion}

Pretibial lacerations typically follow a history of trauma in the elderly and infirmed population. The spectrum of pretibial lacerations are often managed by a variety of healthcare professionals depending on local resource availability. The scarcity of literature in this field and the lack of streamlined management algorithms risks the development of disorganised and archaic treatment with inadequately managed patients presenting later to hospital for surgical management. We believe that surgical intervention should be restricted to more extensive pretibial injuries (Dunkin type III and IV) with senior plastic surgery intervention facilitating accurate referral and management, thereby preventing unnecessary management in the community or by less experienced professionals. If surgery is planned, there should be minimal delay in evacuating the haematoma and correcting lacerations using graft or flap based autologous reconstruction. In-hospital stay should be minimised by pre-emptive discharge planning, early mobilisation and early surgery. It is accepted that prolonged inpatient hospital stay is a risk factor for poor outcomes. ${ }^{18}$ Thus, when conservative management options are appropriate, district nurse care out of hospital may be useful in reducing inpatient stay.

Outcome and mortality data are needed to monitor and audit the management of pretibial lacerations. Randomised controlled trials considering factors such as rate of wound healing, complication rates and quality of life scores assessing physical and mental wellbeing are needed to establish accurate and effective algorithms in the management of these often complex patients. The Dunkin classification provides a solid foundation for management of pretibial lacerations. With type I being treatable in primary care, type II in district general hospitals and type III and IV in tertiary centres, time delay between injury and appropriate treatment will be minimised. Consideration of patient comorbidities should result in early expedition to tertiary centres where appropriate to ensure timely and effective management. Lo et al. ${ }^{15}$ support conservative management but stress that there are no current objective criteria for operative intervention, with the decision residing with the surgeon. They do suggest skin grafting where there is extensive skin loss, gross contamination or conservative management has failed after $2-3$ months. ${ }^{15}$ However, this may result in unnecessary delay in a patient group which is already at high risk of deterioration. We agree that autologous tissue repair should be reserved for extensive injuries (Dunkin III and IV) but believe this should be expedited as first line where clinically warranted.

\section{References}

1. Shuster S, Black MM, McVitie E. The influence of age and sex on skin thickness, skin collagen and density. Br J Dermatol 1975; 93(6): 639-643.

2. Thomson WL, Pujol-Nicolas A, Tahir A et al. A kick in the shins: the financial impact of uncontrolled warfarin use in pre-tibial haematomas. Injury 2014; 45 (1): 250-252

3. Glass GE, Nanchahal J. Why haematomas cause flap failure: an evidence-based paradigm. J Plast Reconstr Aesthet Surg 2012; 65(7): 903-910.

4. Ibuki A, Akase $T$, Nagase $T$ et al. Skin fragility in obese diabetic mice: possible involvement of elevated oxidative stress and upregulation of matrix metalloproteinases. Exp Dermatol 2012; 21(3): 178-183.

5. Harris $\mathrm{CL}$, Fraser C. Malnutrition in the institutionalized elderly: the effects on wound healing. Ostomy Wound Manage 2004; 50(10): 54-63.

6. Tinetti ME, Kumar C. The patient who falls: 'It's always a trade-off'. JAMA 2010; 303(3): 258-266.

7. Cahill KC, Gilleard O, Weir A et al. The epidemiology and mortality of pretibial lacerations. J Plast Reconstr Aesthet Surg 2015; 68(5): 724-728.

8. Creditor MC. Hazards of hospitalization of the elderly. Ann Intern Med 1993; 118(3): 219-223.

9. Dunkin CS, Elfleet D, Ling C et al. A step-by-step guide to classifying and managing pretibial injuries. J Wound Care 2003; 12(3): 109-111.

10. Daabiss M. American Society of Anaesthesiologists physical status classification. Indian J Anaesth 2011; 55(2): 111-115.

11. Tuboku-Metzger V, Chambers J, Osmani $\mathrm{O}$ et al. Early debridement reduces time to healing in elderly patients with pretibial injury. J Plast Reconstr Aesthet Surg 2014; 67(5): 742-744.

12. Karthikeyan GS, Vadodaria S, Stanley PR. Simple and safe treatment of pretibial haematoma in elderly patients. Emerg Med J 2004; 21(1): 69-70.

13. Shankar S, Khoo CT. Lower limb skin loss: simple outpatient management with meshed skin grafts with immediate mobilization. Arch Emerg Med 1987; 4(3): 187-192.

14. Budny PG, Lavelle J, Regan PJ et al. Pretibial injuries in the elderly: a prospective trial of early mobilisation versus bed rest following surgical treatment. Br J Plast Surg 1993; 46(7): 594-598.

15. Lo S, Hallam MJ, Smith S et al. The tertiary management of pretibial lacerations. J Plast Reconstr Aesthet Surg 2012; 65(9): 1,143-1,150.

16. Lamyman MJ, Griffiths D, Davison JA. Delays to the definitive surgical management of pretibial lacerations in the elderly. J Wound Care 2006; 15(9) 422-424.

17. Glass GE, Jain A. Pretibial lacerations: experience from a lower limb trauma centre and systematic review. J Plast Reconstr Aesthet Surg 2014; 67(12): 1,694-1,702.

18. Hirvensalo M, Rantanen T, Heikkinen E. Mobility difficulties and physical activity as predictors of mortality and loss of independence in the communityliving older population. J Am Geriatr Soc 2000; 48(5): 493-498. 